\title{
Analytical approximation of rail bending stress
}

\author{
Piotr Koziol, ${ }^{1, *}$ \\ ${ }^{1}$ Cracow University of Technology, Faculty of Civil Engineering, Kraków, Poland
}

\begin{abstract}
Phenomena associated with railway dynamics are usually analysed by using numerical approaches due to high computational complexity of such systems. However, classical methods based on analytical modelling are still highly valued and desirable by researchers and railway industry. This paper presents analytical solution representing dynamic response of railway track due to moving train in the case of nonlinear foundation. In published papers, one can find analyses of various characteristics such as velocity and acceleration of vibrations of track layers or bending moments of rails. The approach applied in this paper uses the Fourier transform combined with wavelet based approximation applied to the systems of infinitely long beams. The system of Euler-Bernoulli beams resting on viscoelastic foundation represents two-layer model (or one-layer model) of railway track, commonly used in engineering studies. It is shown that although both methods give good results for displacements, analysis of other characteristics, involving derivatives of higher orders, might lead to wrong results, even in the linear case. Possible reasons of this problem are pointed out. Some modifications of the known dynamic railway track models are proposed for further work.
\end{abstract}

\section{Introduction}

Railway track structures can be modelled with a use of many different approaches. FEM based techniques which are widely used in the literature give possibility of discussion of many dynamic effects appearing in rail tracks $[1,2]$. Many published results show, however, that they might be burdened with quite significant error and, additionally, need high computational power. This makes them difficult to apply in many cases, especially in dynamical systems parametrical analysis. Alternative approaches are based on analytical computations, mainly using mathematical formulations, i.e. differential equations with initial and boundary conditions [3, 4]. Such methods allow to analyse desirable features of railway track components, if their description by analytical formulas is possible. Usefulness of such a model depends in turn on ability of introduction of additional assumptions to the system, e.g. nonlinear or stochastic properties. The approximation theory gives reliable tools helping to solve these complex systems [3, $5,6]$. Procedures arising from these techniques are usually effective when one deals only directly with exact solution without further computations involving differentiation or integration [7].

In the literature, one can observe a lack of analytical methods for a proper rail stress evaluation. This paper shows two analytical models of railway tracks: one-layer model and two-layer model. Both these models, although suitable for evaluation of vertical vibrations (or their velocity and acceleration), lead to wrong results with regard to characteristics using derivatives in relation to space variable (e.g. rail bending stress). Question of applicability of the analysed models and methods is partially discussed. Some possible ways of models improvements are mentioned as future work.

\section{Railway track modelled by differential equations}

Two analytical models of railway track are considered in this paper. The one-layer model representing rail vertical vibrations is described by the Euler-Bernoulli beam resting on viscoelastic foundation with nonlinear stiffness. This model is represented by the following equation:

$$
\begin{aligned}
& E I \frac{\partial^{4} y_{r}}{\partial x^{4}}+N \frac{\partial^{2} y_{r}}{\partial x^{2}}+m_{r} \frac{\partial^{2} y_{r}}{\partial t^{2}}+c_{r} \frac{\partial y_{r}}{\partial t} \\
& +k_{r} y_{r}+k_{r N} y_{r}{ }^{3}=q(x, t) .
\end{aligned}
$$

The two-layer model can be described by the following system of two differential equations:

$$
\begin{aligned}
E I \frac{\partial^{4} y_{r}}{\partial x^{4}} & +N \frac{\partial^{2} y_{r}}{\partial x^{2}}+m_{r} \frac{\partial^{2} y_{r}}{\partial t^{2}}+c_{r}\left(\frac{\partial y_{r}}{\partial t}-\frac{\partial y_{s}}{\partial t}\right) \\
& +k_{r}\left(y_{r}-y_{s}\right)-k_{s N} y_{s}^{3}=q(x, t) \\
m_{s} \frac{\partial^{2} y_{s}}{\partial t^{2}} & +c_{s} \frac{\partial y_{s}}{\partial t}+k_{s} y_{s}+k_{s N} y_{s}^{3} \\
& =c_{r}\left(\frac{\partial y_{r}}{\partial t}-\frac{\partial y_{s}}{\partial t}\right)+k_{r}\left(y_{r}-y_{s}\right)
\end{aligned}
$$

The first equation represents rail and the second one describes sleepers layer. The used parameters are: $E I$ $\left[\mathrm{Nm}^{2}\right]$ - bending stiffness of rail steel, $N[\mathrm{~N}]-$ axial force, $m_{r}[\mathrm{~kg} / \mathrm{m}]$ - rail unit mass, $k_{r}\left[\mathrm{~N} / \mathrm{m}^{2}\right]-$ stiffness of

Corresponding author: pkoziol@pk.edu.pl 
fasteners, $c_{r}\left[\mathrm{Ns} / \mathrm{m}^{2}\right]$ - viscous damping of fasteners, $m_{s}$ $[\mathrm{kg} / \mathrm{m}]$ - unit mass of sleepers uniformly distributed along the track. Effect of sleepers bending is neglected. The two parameters: $k_{s}\left[\mathrm{~N} / \mathrm{m}^{2}\right]$ and $c_{s}\left[\mathrm{Ns} / \mathrm{m}^{2}\right]$ denote stiffness and viscous damping of sleepers foundation, respectively. Geometrical imperfections of rolling surface of the rail head are represented by regular cosine wave with frequency $\Omega[\mathrm{rad} / \mathrm{m}]$ and amplitude $s_{0}[\mathrm{~m}]$. The load $q[\mathrm{~N} / \mathrm{m}]$ is considered as a set of distributed forces produced by wheels of train and associated with train axles configuration. In the case of constant speed $v$ $[\mathrm{km} / \mathrm{h}]$, geometrical imperfections produce the varying forces with circular frequency $\omega=\Omega v$ and the amplitude $F_{s}=s_{0} k_{c}$. These forces are distributed on the wheel-rail contact area and the contact is modelled by a single spring $k_{c}$. The terms $k_{r N} y_{r}{ }^{3}$ (eq. (1)) and $k_{s N} y_{s}^{3}$ (eq. (2)) represent nonlinear factor associated with nonlinear part of rail foundation stiffness $k_{r N}\left[\mathrm{~N} / \mathrm{m}^{4}\right]$ and rail track foundation stiffness $k_{s N}\left[\mathrm{~N} / \mathrm{m}^{4}\right]$, respectively $[6,8,9]$.

One can apply the moving coordinate system $(\eta=x, \xi=x$ $v t$ ) to equations (1-2) and then these formulas can be transformed to the following form:

$$
\begin{aligned}
& E I \frac{\partial^{4} y_{r}}{\partial \xi^{4}}+N \frac{\partial^{2} y_{r}}{\partial \xi^{2}}+m_{r}\left(\frac{\partial^{2} y_{r}}{\partial t^{2}}-2 v \frac{\partial^{2} y_{r}}{\partial t \partial \xi}\right. \\
& \left.+v^{2} \frac{\partial^{2} y_{r}}{\partial \xi^{2}}\right)+c_{r}\left(\frac{\partial y_{r}}{\partial t}-v \frac{\partial y_{r}}{\partial \xi}\right)+k_{r} y_{r} \\
& +k_{r N} y_{r}^{3}=q(\xi, t)
\end{aligned}
$$

and

$$
\begin{aligned}
& E I \frac{\partial^{4} y_{r}}{\partial \xi^{4}}+N \frac{\partial^{2} y_{r}}{\partial \xi^{2}} \\
& +m_{r}\left(\frac{\partial^{2} y_{r}}{\partial t^{2}}-2 v \frac{\partial^{2} y_{r}}{\partial t \partial \xi}+v^{2} \frac{\partial^{2} y_{r}}{\partial \xi^{2}}\right) \\
& +c_{r}\left(\frac{\partial y_{r}}{\partial t}-v \frac{\partial y_{r}}{\partial \xi}-\frac{\partial y_{p}}{\partial t}+v \frac{\partial y_{s}}{\partial \xi}\right) \\
& +k_{r}\left(y_{r}-y_{s}\right)-k_{s N} y_{s}^{3}=q(\xi, t) \\
& m_{s}\left(\frac{\partial^{2} y_{s}}{\partial t^{2}}-2 v \frac{\partial^{2} y_{s}}{\partial t \partial \xi}+v^{2} \frac{\partial^{2} y_{s}}{\partial \xi^{2}}\right) \\
& +c_{s}\left(\frac{\partial y_{r}}{\partial t}-\frac{\partial y_{s}}{\partial \xi}\right)+k_{s} y_{s} \\
& -c_{r}\left(\frac{\partial y_{r}}{\partial t}-v \frac{\partial y_{r}}{\partial \xi}-\frac{\partial y_{s}}{\partial t}+v \frac{\partial y_{s}}{\partial \xi}\right) \\
& -k_{r}\left(y_{r}-y_{s}\right)+k_{s N} y_{s}^{3}=0
\end{aligned}
$$

These models can be solved by applying either the Fourier series or the Fourier transform, depending on assumptions with regard to the E-B beams and the decision about the nonlinearity investigation.

The load representing moving train can be described as follows:

$$
\begin{aligned}
& q(\xi, t)=P_{S}(\xi, t)+P_{D}(\xi, t) \\
& =\sum_{l=0}^{l_{T}}\left(\frac{P_{0}}{a}+\frac{P_{1}}{a} e^{i \omega t}\right) \cos ^{2}\left(\frac{\pi\left(\xi-s_{l}\right)}{2 a}\right) H\left(a^{2}-\left(\xi-s_{l}\right)^{2}\right)
\end{aligned}
$$

where

$$
\begin{aligned}
& P_{S}(\xi, t) \\
& =\sum_{l=0}^{l_{T}} \frac{P_{0}}{a} \cos ^{2}\left(\frac{\pi\left(\xi-s_{l}\right)}{2 a}\right) H\left(a^{2}-\left(\xi-s_{l}\right)^{2}\right)
\end{aligned}
$$

is the stationary part of load, i.e. constant in time, and

$$
\begin{aligned}
& P_{D}(\tilde{x}, t) \\
& =\sum_{l=0}^{l_{T}} \frac{P_{1}}{a} e^{i \omega t} \cos ^{2}\left(\frac{\pi\left(\xi-s_{l}\right)}{2 a}\right) H\left(a^{2}-\left(\xi-s_{l}\right)^{2}\right)
\end{aligned}
$$

represents the part of load varying in time, produced by rail head surface imperfections of cosine form and constant amplitude. $H(),. 2 a, s_{l}, l_{T}, \omega$ and $V$ are the Heaviside function, the span of each load related to single wheel, the distance of consecutive forces produced by wheels, the number of axles, the frequency and the velocity of the moving load, respectively. The axles configuration can be also introduced by inclusion of the phase shift associated with the wheel position on the cosine shape of irregularity $[3,10]$.

\section{Solution}

The following method of solution can be applied to both systems, (3) and (4a-4b), in the case of infinitely long beams. It deals also with the assumed nonlinearity of foundation stiffness.

The cubic term representing nonlinearity can be expanded by Adomian polynomials $[5,6,10]$ :

$$
\begin{aligned}
& y^{3}(x)=\sum_{j=0}^{\infty} Q_{j}(x) \\
& =\sum_{j=0}^{\infty} \frac{1}{j !}\left[\frac{d^{j}}{d s^{j}}\left(\sum_{k=0}^{\infty} \lambda^{k} y_{k}(x)\right)^{3}\right]_{s=0}
\end{aligned}
$$

Both models, the one-layer and the two-layer can be solved by applying the Fourier transform

$$
\tilde{y}(\omega)=F[y(\xi)]=\int_{-\infty}^{\infty} y(\xi) e^{-i \omega \xi} d \xi
$$

Because the form of solution in the transform domain becomes quite complicated, classical methods of retransformation are inefficient when applied to parametrical analysis. The response in the physical domain can be effectively obtained from the transform domain by applying the coiflet based approximation of the inverse Fourier transform $[5,6,10]$ : 


$$
\begin{aligned}
& y(\xi)=\lim _{n \rightarrow \infty} y_{n}(\xi) \\
&=\lim _{n \rightarrow \infty} \frac{1}{2^{n+1} \pi} \prod_{k=1}^{k p}\left(\sum_{j=0}^{3 N-1} p_{j} e^{i j \xi / 2^{n+k}}\right) \\
& \\
& \qquad \sum_{k=-\infty}^{+\infty} \tilde{y}\left(\left(k+\sum_{k=0}^{3 N-1} k p_{k}\right) 2^{-n}\right) e^{i \xi k 2^{-n}}
\end{aligned}
$$

where $N$ is the degree of the used coiflet filter $\left(p_{k}\right)$ and $k_{p}$ is a number evaluated on the basis of stabilization of the obtained solution $[5,6,11]$.

\section{Examples and discussion}

In the case of rail and sleepers displacement, one can find sufficiently exact solutions by applying the technique described in section 3. These solutions can be successfully used in parametrical analysis when one takes into account engineering applications. They have been also verified by experimental measurements $[10$, 12]. In the case of stresses produced by dynamic excitations, the problem of analytical modelling becomes more difficult.

The following system of parameters is taken in preparation of examples:

$E I=6.4 * 10^{6} \mathrm{Nm}^{2}, N=0, m_{r}=60 \mathrm{~kg} / \mathrm{m}, k_{r}=8.8^{*} 10^{7}$ $\mathrm{N} / \mathrm{m}^{2}, c_{r}=3950 \mathrm{Ns} / \mathrm{m}^{2}, m_{s}=267 \mathrm{~kg} / \mathrm{m}, k_{s}=8.5^{*} 10^{7}$ $\mathrm{N} / \mathrm{m}^{2}, c_{s}=8.2 * 10^{3} \mathrm{Ns} / \mathrm{m}^{2}$ and $v=150 \mathrm{~km} / \mathrm{h}$. The nonlinear stiffness coefficients $k_{r N}$ and $k_{s N}$ can be taken similarly to values considered in past publications $[6,10-$ 12], although it is neglected in this paper for calculation of normal bending stress. It is assumed that the part of load $P_{D}$ (see eq. $(6 \mathrm{~b})$ ) is related to the rail deflection between sleepers and there are no other imperfections on rolling surface of rail head. The axles configuration of fast train Pendolino EMU250 is also considered.

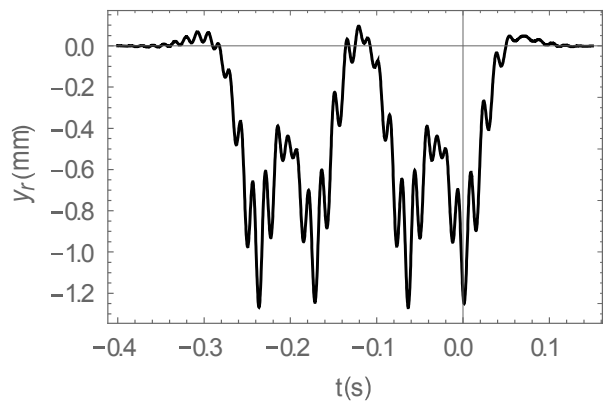

Fig. 1. Vertical vibrations of rail.

Figure 1 presents the dynamic response of railway track to moving train in terms of vertical displacements of rails obtained analytically by using the two-layer model and the coiflet based technique in the case of linear foundation stiffness.

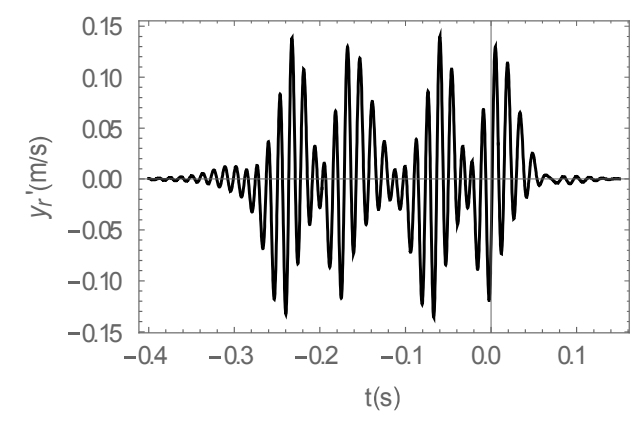

Fig. 2. Velocity of rail vertical vibrations.

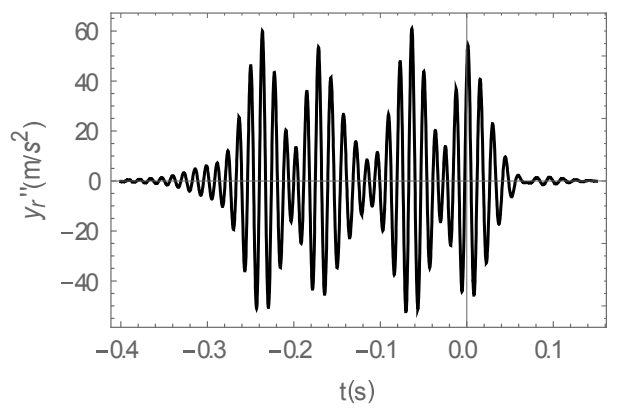

Fig. 3. Vertical vibrations acceleration.

Figures 2-3 show vibration velocity and vibration acceleration for the same system of parameters. As mentioned before, the results for vertical vibrations have been verified experimentally $[10,12]$. When one deals with vibrations velocity or acceleration, the obtained results need to be verified by experimental measurements or other methods of solution for extended range of parameters, although maximal values are close to real ones, at least in the case of the track in relatively good condition. Some doubts arise from the analysis of the normal bending stresses, involving derivatives of higher order with respect to the space variable. It can be observed that analytically obtained results significantly exceed limit values of the strength of rail steel R260 (around $800 \mathrm{MPa}$ ).

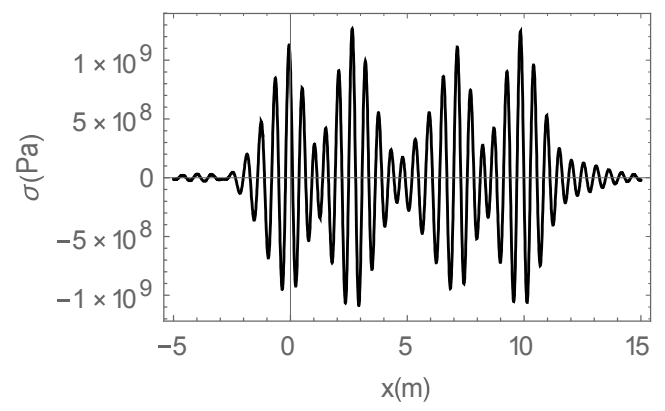

Fig. 4. Distribution of normal stress in rail obtained by using two-layer model and wavelet approximation.

Figure 4 presents the normal stress distribution in rail with irregularity related to the sleeper spacing, showing the method ability to analytically calculate stresses. 


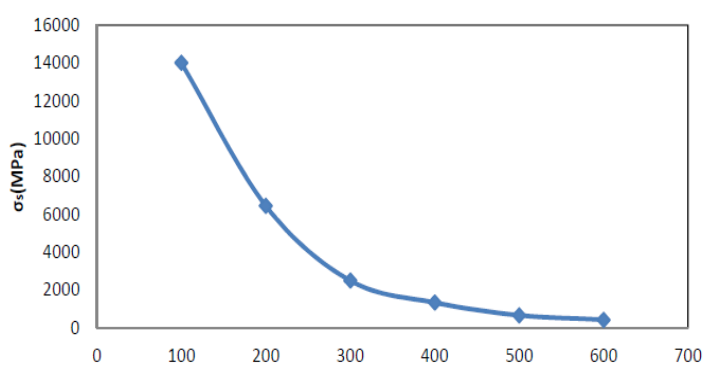

Fig. 5. Maximal normal stress in rail axis depending on the length [mm] of cosine imperfection [7].

For some lengths of waves, associated with the considered type of irregularity appearing on rail head rolling surface, the maximal value of the normal stress can significantly exceed the limit value (a few hundred percent and more). For example, for the length of irregularity equal to $30 \mathrm{~cm}$ (cosine type of imperfection), which is twice less than the distance between sleepers, and the train speed $\mathrm{V}=200 \mathrm{~km} / \mathrm{h}$, the obtained maximal value of the normal stress in rail axis can be estimated around $1500 \mathrm{MPa}$. This value is obtained under assumption that the force generated by the considered irregularity is the same as for a length of $60 \mathrm{~cm}$, which corresponds to the sleepers spacing. In reality, this additional dynamic force can be stronger and therefore the resulting normal stress should reach higher values. Figure 5 shows maximal normal stress in rail axis depending on the length $[\mathrm{mm}]$ of cosine imperfection, under described above assumption about forces.

The project [7] was aimed at showing examples of analytically derived stresses exceeding limit values when using models validated and known from the literature. The analysis was carried out for a wide range of physical parameters and train speeds showing that a lack of appropriate methods (models) for the normal stress calculation should be underlined.

\section{Conclusions}

One can observe that the obtained results for normal bending stress in rail axis exceed, for some systems of parameters, limit values independently of model and method of analytical solution. One can say that applied approaches (models and/or methods) are insufficient for stresses analysis. In order to improve this analysis, one might suggest consideration of additional mechanical features of the investigated systems. These features remain unrecognised so far. In future, they should lead to reformulation of railway track models. Among them, one should mention a more detailed approach to rail modelling. This modification should avoid treating the rail as a homogeneous beam. Some preliminary results are obtained for "head on web" problem or rail internal damping included in the dynamic beam equation. These results are valid for some specific range of parameters only. They are especially strongly dependent on the load frequency. Therefore the undertaken analysis is not satisfactory and should be extended. One should underline that all analytical models and the wavelet based method considered in this paper are valid for rails and sleepers vertical displacement analysis.

\section{References}

1. D. Tran, Adv. Struct. Eng., 12, 3, 399-409 (2009)

2. P. Amado-Mendes, P.A. Costa, L.M.C. Godinho, P. Lopes, Eng. Struct., 104, 141-154 (2015)

3. W. Czyczula, P. Koziol, D. Kudla, S. Lisowski, J. Vib. Control., 23, 18, 2989-3006 (2017)

4. P. Koziol, Proceedings of ICoEV2015, International Conference on Engineering Vibration, Ljubljana, Slovenia, 7-10 September 2015, ISBN 978-9616536-97-4, paper 273, (2015)

5. P. Koziol, VDM Verlag Dr. Müller, Saarbrucken, (2010)

6. P. Koziol, J. Theor. Appl. Mech., 52, 3, 687-697 (2014)

7. P. Bulinski, MSc thesis (in Polish), Cracow University of Technology (2016)

8. T. Dahlberg, Proceedings of Fifth International Conference on Structural Dynamics, Munich (2002)

9. M.H. Kargarnovin, D. Younesian, D.J. Thompson, C.J.C. Jones, Comput. Struct., 83, 1865-1877 (2005)

10. P. Koziol, Mech. Syst. Signal Process., 79, 174-181, (2016)

11. P. Koziol, Z. Hryniewicz, Shock Vib., 19, 995-1007, (2012)

12. P. Koziol, Proceedings of the Fifteenth International Conference on Civil, Structural and Environmental Engineering Computing, Civil-Comp Press, Stirlingshire, UK, Paper 199 (2015) 